\title{
A qualitative exploration of supports and unmet needs of diverse young women with breast cancer
}

\author{
Kathryn J Ruddy, MD MPH, ${ }^{a}$ Mary L Greaney, PhD, ${ }^{\mathrm{b}}$ Kim Sprunck-Harrild, MSW, MPH, \\ Meghan E Meyer, BS, ${ }^{d}$ Karen M Emmons, PhD, ${ }^{c}$ and Ann H Partridge, MD, $\mathrm{MPH}^{\mathrm{d}}$
}

${ }^{a}$ Department of Oncology, Mayo Clinic, Rochester, Minnesota; ${ }^{b}$ Department of Kinesiology, University of Rhode Island, Kingston, Rhode Island, and ${ }^{\mathrm{c} C e n t e r}$ for Community-Based Research and dDepartment of Medical Oncology, Dana-Farber Cancer Institute, Boston, Massachusetts

Background Young women with breast cancer face different challenges than those faced by older women because of their age and life stage, yet few studies have focused on the different challenges faced by women from diverse populations.

Objective To explore existing supports that are important during diagnosis and treatment and the unmet needs for information and support in young women with breast cancer.

Methods We conducted 20 semistructured interviews in English with women aged 42 or younger who had been diagnosed with stage I-III invasive breast cancer within the previous 4 years. We recorded and transcribed the interviews and used collaborative group immersion/crystallization to analyze data, identify emergent themes, and determine if there were differences by race/ethnicity.

Results 20 participants, recruited from 9 US states and Canada, were interviewed, of whom 25\% were Hispanic, 15\% were black, $50 \%$ were white and non-Hispanic, and $10 \%$ were another race/ethnicity. Faith and/or spirituality and family were reported as important sources of support by many of the participants. Most of them lamented the inadequacy of their connections with other young survivors and also of supports for their family. Some recommended that young patients be provided with more information about: treatment-related physical and emotional changes; fertility and menopause; relationships after cancer; navigating work challenges; and transitioning into survivorship. None of these supports or recommendations was limited to a specific race/ethnicity or geographic region.

Limitations Small sample size, exclusion of non-English speakers.

Conclusions Key informant interviews of young breast cancer survivors identified similar needs for education and support across various races/ethnicities and geographies.

Funding/sponsorship Supported by an ASCO Cancer Foundation/Susan G Komen for the Cure Improving Cancer Care Grant (PI: Partridge) and by NIH 5K05 CA124415-05 (PI: K Emmons).

$\Lambda$ n estimated 232,340 women were diagnosed with breast cancer in the United States in 2013, and although the median age at diagnosis is $61,{ }^{1}$ about $11 \%$ of them were younger than 45 years. Although breast cancer is most common in white women, breast cancers in black women are more likely to occur at a young age and are more frequently fatal; both white and black women more commonly develop and die from breast cancer than do Hispanic women. ${ }^{1}$ Few studies have focused on identifying the emotional and educational needs of young nonwhite patients. The Centers for Disease Control and Prevention recently recognized survivorship issues in young breast cancer patients as an important focus for research, ${ }^{2}$ and previous research suggests that nonwhite, non-Hispanic survivors of mixed ages may have unique needs. ${ }^{3-6}$ One study $(\mathrm{n}=82)$ found that younger age was a predictor of anxiety and depression in black patients, ${ }^{7}$ suggesting that supportive interventions may be particularly important in this group.

We previously conducted focus groups with young breast cancer survivors based in Boston, Massachusetts ( $\mathrm{n}=36$ ), and identified the following 3 key issues in this relatively homogenous population: feelings of isolation and unique challenges because of their young age; difficulties transitioning into the survivorship period; and desire for additional supports and information to meet their needs. ${ }^{8}$ The majority of women in that study were white and non-Hispanic, so we conducted in-depth interviews with a racially/ethnically and geograph-

Accepted for publication November 13, 2014. Correspondence: Kathryn J Ruddy, MD MPH; ruddy.kathryn@mayo.edu. Disclosures: The authors have no disclosures. JCSO 2015;13:323-329. (2015 Frontline Medical Communications. DOI 10.12788/jicso.0169. 
ically diverse sample of young women with a history of breast cancer to inform a multicenter intervention study for young breast cancer patients by identifying other potentially important issues and exploring the generalizability of our previous findings, and to explore possible differences by race/ethnicity and region.

\section{Methods}

\section{Recruitment}

We asked 18 geographically dispersed North American hospitals and organizations that provide care to diverse populations of breast cancer patients to distribute study flyers during April-November 2011 calling for potential participants to contact our study staff. The flyers included a synopsis of the study and contact information (e-mail address/telephone number). When the study staff were contacted, they answered questions about the study and confirmed the callers' eligibility criteria, which included: age 18-42 years at diagnosis with a stage I-III breast cancer based on self-report within the previous 4 years; having no known metastatic disease; and being able to speak English. Sample size was preset at 20 based on financial constraints with the study funding. We required that at least 10 of the participants be nonwhite or Hispanic, but otherwise the first 20 eligible callers were enrolled in the study.

\section{Key informant enrollment and interview procedures}

Once a patient's eligibility was established, an interview was scheduled. We did not confirm diagnosis or disease status other than by self-report. Telephone interviews were conducted by a single staff member trained in qualitative research methods using a semistructured interview guide with 5 core open-ended questions and subsequent follow-up questions based on participant responses. The guide was developed using an ecological model, and was designed to explore existing supports during diagnosis and treatment and unmet needs for information and support. The interviews occurred during May-November 2011. After a participant gave consent, she completed a brief demographic survey. Interviews lasted about an hour and were audio-recorded. All of the participants received a $\$ 25$ (US currency) gift card. This protocol was approved by the institutional review board at Dana-Farber Cancer Institute.

\section{Analysis}

All audio-recordings were professionally transcribed, and identifiers were removed. We used a collaborative process of immersion/crystallization to analyze the qualitative data. ${ }^{9}$ Through an iterative process, 2 researchers trained in qualitative research methods independently read each transcript and reflected on the data while taking detailed notes. They then met to discuss and iden- tify emerging themes. After several cycles of immersion and reflection, they developed a codebook, which was used to code all transcripts. The researchers met regularly to discuss coded transcripts and identified themes and assessed if there were differences by race/ethnicity (white or other) or geography. Frequencies and means for the demographic data were obtained using SAS version 9.3 (SAS Institute Inc, Cary, NC, USA).

\section{Results}

\section{Participants}

Twenty women participated in the study; the mean age was 35.9 years (SD, 5.0; range, 26-42). The sample was racially and ethnically diverse: $25 \%$ identified as Hispanic, $15 \%$ as black, $50 \%$ as white and non-Hispanic, and $10 \%$ as another race. Four lived in Texas, 3 in Pennsylvania, and 2 in each in Massachusetts, New York, South Dakota, Tennessee, and Wisconsin. One woman lived in California, and another in Ontario, Canada. Seventy-five percent were employed at the time of diagnosis, 2 were stay-at-home mothers, and 2 were unemployed. Nearly half reported a household annual income of more than $\$ 75,000$, and $75 \%$ reported that they had enough money to pay the bills without cutting back on other expenses (Table).

\section{Supports and needs identified}

The themes that emerged during analyses ( 3 existing supports, 5 issues about which they felt they had been inadequately informed, and 2 additional unmet needs) are presented here with exemplar quotes. No clear differences in existing or needed supports by race/ethnicity (white vs nonwhite) were identified.

\section{Existing supports during diagnosis and treatment} Relationships. Almost all of the participants reported that spousal/partner and familial support was instrumental to their ability to navigate their cancer journey. One participant explained,

\footnotetext{
My mother and ... father ... were my rock ... there were ... good days and bad days but ... I couldn't have passed 'em all without what my family did for me.
}

Supportive work environments. Most employed participants spoke of work environments that allowed for flexible schedules and/or reduced workloads at critical times as being important in allowing them to continue to work. One person noted,

My boss was great ... If I wasn't feeling up to doing, like, all of my tasks, I could just fill in and help here and there.

Faith and/or spirituality. These were identified as other 
TABLE Patient, tumor, and treatment characteristics

\section{Characteristic}

Race/ethnicity, n (\%)

White, non-Hispanic $\quad 10(50)$

Hispanic 5 (25)

Black 3 (15)

Other (native Hawaiian, Caribbean) 2 (10)

Mean age, y (SD; range) $\quad 35.9$

15.03 ;

26.0-42.0)

$\begin{array}{ll}\text { Age at diagnosis, } n(\%) & \\ 26-29 y & 2(10) \\ 30-34 y & 7(35) \\ 35-39 y & 6(30) \\ 40-42 y & 5(5)\end{array}$

Education, n (\%)

High-school graduate/GED 2 (10)

Some college/2-year degree $3(40)$

College graduate $10(50)$

$\begin{array}{ll}\text { Married or living as married, } n(\%) & 12(60) \\ \text { Have child/children, } n(\%) & 12(60) \\ \text { Household income, } n(\%) & \\ <\$ 25,000 & 4(20) \\ \$ 25,000 \text { to }<\$ 50,000 & 3(15) \\ \$ 50,000 \text { to }<\$ 7,000 & 4(20) \\ >\$ 5,000 & 9(45)\end{array}$

Perceived household financial situation, $\mathrm{n}(\%)$

Have enough money to pay the bills, and to get special things

Have enough money to pay the bills, but little spare money to buy extra or special things

Enough money to pay the bills, but only because have cut back on things

Have difficulty paying the bills, no matter what you do

2 (10)

Health insurance, $n(\%)$

Have health insurance

Insurance covered all treatment costs

$11(55)$

Disease stage, $\mathrm{n}(\%)$

I $7(35)$

II

III

Not sure

2 (10)

Type of treatment, $\mathrm{n}(\%)$

Mastectomy

$13(65)$

Reconstruction

$9(45)$

Chemotherapy

$16(80)$

Radiation

$10(50)$

Hormonal therapy (current or past) stand out sources of support for nearly half of the women, helping them accept the diagnosis, cope with fears, and find strength during treatment. One woman explained,

\author{
I had to just keep with my faith ... God will help me get \\ better ... for me, number one was ... my faith in God.
}

\section{Needed information and supports}

Side effects of treatment. Surgical changes: Unilateral or bilateral mastectomy was reported by $65 \%$ of participants, and $69 \%$ of them had reconstructive surgery. Many felt underprepared for this long, often multiphase process, and some expressed that their expectations and feelings were out of sync with the care they received. One woman explained,

\begin{abstract}
$\mathrm{He}$ [my surgeon] ... often spoke to me from a place of the end result ... there were times I felt very frustrated because things were often relayed to me from this final vantage point.
\end{abstract}

Many women who had mastectomy reported that the loss of their breast or breasts was one of the most difficult parts of their treatment, and chose reconstruction surgery to help mitigate this loss. However, $40 \%$ of those who had reconstruction were unhappy with the results due to lack of symmetry, scarring, and differences in breast size/shape. Several women reported that they no longer felt like themselves and/or they became self-conscious about their bodies.

Toxicities of chemotherapy: Some side effects of chemotherapy were unanticipated by participants, who felt they had not be adequately educated about possible symptoms (eg, mouth sores, bone pain) and how to manage them. They stated that additional upfront information could have reduced their anxieties. Several women voiced displeasure that they were unprepared for chemo brain, the temporary inability to remember things or find words to express themselves. One stated,

\footnotetext{
I think it would have been nice ... to know that the potential for it [chemo brain] exists? So that when it happens, you don't think you've completely lost your mind.
}

Another difficult side effect was unexpected weight gain; many wrongly had assumed that they would lose weight. One woman said,

$$
\begin{aligned}
& \text { I can put on make-up and I can buy wigs ... but the weight } \\
& \text { gain's just ... I would ... complain and complain. }
\end{aligned}
$$

Menopause, sexual functioning, and fertility. Menopause and sexual functioning: About half of the participants felt that early menopause and changes in sexual functioning 
had not been addressed completely, and had desired more information about how to increase libido and reduce vaginal dryness. Some women turned to other sources (eg, friends, internet) because their medical providers were not informative enough. One explained,

Did I get enough information? No, they pretty much just directed me to go look at a couple of web sites ... I had to start doing research on my own, and come back to her [my oncologist] with questions.

Fertility: Almost half of participants spoke of wanting to have children or have another child. Many did not feel that their medical providers discussed the effect of treatment on fertility and fertility-related concerns thoroughly enough before a treatment plan had been set. As one participant explained,

It's very clear that my oncologist is treating my cancer. She's not interested in my fertility issue.

Moreover, one woman mourned the repercussions of her inadequate pre-treatment fertility discussion, saying she

... would have done things differently ... I didn't do anything with saving eggs or anything, so now, my chances are slim ... looking back I wish I would have thought about that.

Guidance on navigating current and future romantic relationships. A number of participants expressed the opinion that women need more information about how a cancer diagnosis may negatively affect intimate relationships. One woman said,

It almost brought it to a point of getting a divorce ... and we both weren't ... prepared for that.

Single women often voiced concerns about dating. They wanted information about how and when to discuss their cancer histories with potential new partners. A participant said,

I haven't found a support mechanism for that [dating] ... I'm dealing with the depression. How do you tell someone? Or that I have a scar that looks like a zip line that goes all the way across my body ... [I] kind of need to be in a support group with young people who are going through this dating process or trying to get back into the dating process.

Information pertaining to work. Many employed participants said that more information about how treatment would affect their ability to work could have been beneficial. They would have liked to know how to speak with employers about their diagnoses and treatment. A few women who believed they had been terminated due to the impact that treatment had on their ability to work were particularly interested in employment-related issues. One woman stated,

I just found out about the American Disability Act and everything that goes along with that, and I ... feel like if I would have known about that, earlier, maybe I could have ... prepare[d] for all this stuff and make sure I had everything together in case ... this [loss of job] came about.

Information about survivorship. All study participants were finished with their active cancer treatments (other than hormonal therapy), and most felt they had transitioned relatively smoothly into survivorship. However, several spoke of the need for more preparation for this transition. One stated,

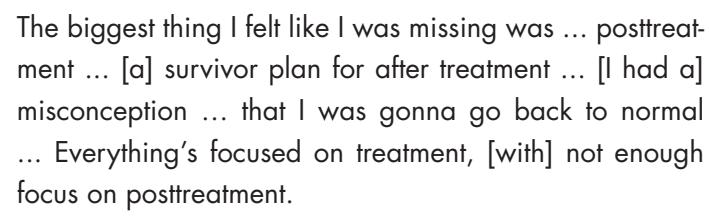

Connections with other young breast cancer survivors. Many participants, regardless of race/ethnicity, reported feeling isolated from other women with breast cancer because of their young age. About half of them participated in organized cancer patient gatherings (eg, support groups), but the gatherings were generally not specific to young women. Only 2 women spoke of being involved in a coalition for young survivors, and this involvement began long after their diagnoses; many others said they would have benefited from this type of connection. As one woman explained,

I really think that they need to have a support group for younger women. Because the support group that we have ... all seem to be older, and they all like to do crochet ... But I wanted to live ... to get out ... to go kayaking. I want to go do something that I've never... done before .... My friend that died at 34 ... we wanted to start a group of younger survivors and, and create some adventure trips ... I want to live my life to [do] ... what makes me happy!

Participants felt that connections with other young breast cancer patients would have allowed for a more informative discussion about body image, dating, relationships, sexual functioning, and making surgical decisions. One participant explained there are 
... certain things that we could talk about, compared to older people that might not be at the same stage in our life. I don't think they will understand as well as somebody my age.

In addition, at least one participant felt that it would have been extremely useful to be matched with someone of a similar race and body type because

... we don't have the same body shape. So, what a white woman would go through, she may be happy with the fact that she's coming up to a D [bra size]. But I'm going down!

Supports for partner/family members. Participants spoke of wanting information and written materials to share with their partners/spouses so that they would better understand what they were going through. In addition, women would have liked their caregivers to have been offered counseling and/or support groups. As one woman said,

There's not any [support group] ... for spouses, and I think that would have been very helpful ... he definitely needed an outlet to ... express ... emotions.

Another woman noted,

\begin{abstract}
My mom took it hard ... I had to convince her every day that I wasn't dying ... that pretty much broke her heart ... you spend more time making them feel better than making you feel better.
\end{abstract}

She also said she would have liked more guidance on how to have told her mother:

Maybe I did something wrong? ... maybe there was a better way for me ... to make her feel more secure.

\section{Discussion}

This study evaluated sources of support and unmet needs of a diverse sample of young breast cancer patients. Many of the themes identified in this study are similar to those that we have previously identified in focus groups comprised of predominantly non-Hispanic white breast cancer survivors in Massachusetts. ${ }^{8}$ The similarities of data generated by these 2 qualitative studies may in part reflect that half of those in the current study were non-Hispanic white women.

Because spousal, familial, and work-based supports are consistently credited as important to young cancer patients, health care providers might wish to ask them more routinely about their social networks and the abil- ity of those networks to provide emotional and tangible support. If those supports are not sufficient, actively connecting patients with other young patients, referring them to survivor groups, and counseling by a social worker or psychologist may be helpful. Given the unique challenges of dating and sustaining romantic relationships while undergoing breast cancer treatment, it may be useful to ask patients about these aspects of their lives, and to facilitate connections between patients who have similar relationship statuses. Also, patients with less accommodating work environments might benefit from tips on how to communicate their needs at work.

It is interesting to note that faith and spirituality were more frequently mentioned as existing sources of support than in our previous Boston-based focus groups study. It is possible that women felt more comfortable discussing these issues during telephone-based individual interviews than with other survivors present. Alternatively, women from other parts of the country or with a Hispanic or nonwhite race/ethnicity might be more likely to derive strength from faith and spirituality. Other studies have shown that faith and spirituality help cancer patients, though most existing literature has focused on older populations. ${ }^{10-12}$ Nonetheless, a recent qualitative study of young African American breast cancer survivors $(n=33)$ found that $21 \%$ identified God or their church as their primary source of support. ${ }^{13}$ There are earlier data supporting the importance of spirituality and the use of faith-based interventions for African American and Hispanic cancer survivors. ${ }^{14-17}$ However, we did not find that reliance on religion or spirituality was limited to a specific race or ethnicity.

Similar to other studies, we identified that young survivors desired more information about the toxicities and mixed outcomes of surgery and chemotherapy. A qualitative study that focused on women who were undergoing implant breast reconstruction ( $\mathrm{n}=28$, no race/ethnicity data provided) found that many had false expectations (eg, that breasts would look natural) and that those who had more inaccurate expectations experienced higher levels of dissatisfaction with the results. ${ }^{18}$ Patient education and counseling prior to surgery have been shown to improve satisfaction, ${ }^{19}$ which suggests that young women with breast cancer might benefit from robust preoperative counseling about variable outcomes, especially for those who choose mastectomies over lumpectomies ${ }^{20}$ even though there is no clear survival advantage with mastectomy. ${ }^{21}$

Previous studies with more homogeneous populations than the current study have demonstrated that early menopause, sexual dysfunction, and infertility were of significant concern to young breast cancer patients. ${ }^{22-24}$ Regardless of race/ethnicity, young women seem to be particularly frustrated by the inadequacy of the pretreatment education they receive from their providers regarding fertility. 
A qualitative study $(\mathrm{n}=18)$ found that young (age range, 19-42 years) cancer survivors (no race/ethnicity data provided) relied on doctors to initiate conversations about fertility because they did not feel comfortable doing so. ${ }^{25} \mathrm{In}$ another qualitative study, young breast cancer survivors ( $\mathrm{n}$ $=10$ ) explained that they preferred that providers discuss fertility early and not make assumptions regarding their desires. ${ }^{26}$ Results of our current study confirm these findings and emphasize the importance of proactive discussion by oncologists about fertility concerns in a diverse population, as recommended by the American Society of Clinical Oncology (ASCO). ${ }^{27,28}$

The median age of breast cancer diagnosis is 61 years, ${ }^{1}$ which also is the average retirement age ${ }^{29}$ so the impact of treatment on work may not be a concern for half of all breast cancer patients. Therefore, health care providers may not routinely discuss treatment scheduling and the impact of side effects on a patient's work situation. Our findings suggest that younger, employed women could benefit from more attention to their employment status and job requirements.

As in our previous study with a less diverse sample of participants, transitioning into survivorship care was identified as a significant challenge for these young women. Treatment summaries and survivorship care plans may help young breast cancer patients from various communities better understand what is behind and ahead of them.

Additional research is needed to evaluate the value of connecting young patients to others who resemble them in age and life stage (as suggested by our prior work), as well as resemble them in other factors such as cultural background (as suggested by 1 participant in the current study). Racial/ethnic minority women may feel especially isolated in the face of a breast cancer diagnosis at a young age, and may benefit greatly from meeting other similar patients. In small or rural oncology practices that are not able to arrange these connections amongst their patients, providers may wish to refer patients to non-profit organizations such as the American Cancer Society's Reach to Recovery program (www.cancer.org) and Imerman Angels (www.imermanangels.org), both of which link a cancer patient with another person who survived the same type of cancer, when possible matching them by stage and age. Online communities for young patients with cancer (eg, www.stupidcancer.org) may also help meet these needs.

As in our prior focus group study of young breast cancer survivors, ${ }^{8}$ we found that this more diverse sample also expressed a need for supportive services for their loved ones (spouse/partner, children, parents). The Institute of Medicine has reported mixed results on this topic (with some studies showing improved coping/confidence and reduced distress, but others not) when supportive services were made available to caregivers (eg, support groups, psy- chotherapy, and respite care services $)^{30}$ and stressed that caretakers' abilities and limitations should be evaluated to ensure that they are capable of providing the necessary care. Several existing measures are available to assess the needs of family members of cancer patients, but these are not yet widely used in oncology clinics. ${ }^{31-33}$ For the family members of young breast cancer patients, more research is needed to determine what types of support would be most beneficial.

Limitations of this work include the small sample size and the exclusion of non-English speakers, both of which restrict generalizability. Future research should collect data regarding the types of jobs and the types of insurance held by participants, because both of those may have a substantial impact on the results, and neither was part of the demographic survey in this study. Furthermore, generalizability is limited by the fact that most participants were well educated and relatively affluent. We hope that future studies will be able to attract more economically and educationally disadvantaged populations to confirm these findings and/or identify their unique needs. Also, we hope to assess which health care providers may be best equipped to meet these needs, and what system-based changes (eg, starting to offer patient navigators and/or survivor mentors to all young patients) could be implemented to improve quality of life for survivors and their loved ones.

\section{Conclusion}

Young women with breast cancer seem to have similar needs and preferences across the country, not specific to a given race/ethnicity. A large proportion of the women in this relatively diverse sample reported finding support in their faith and/or spirituality. These results were incorporated into materials that are currently being distributed to patients and oncologists as part of the Young \& Strong Study, a large randomized trial of an educational intervention designed to improve care of young women with early stage breast cancer.

\footnotetext{
Acknowledgments

The authors thank the women who participated in the key informant interviews, as well as the institutions and organizations that assisted with recruitment: Tracy Battaglia (Boston Medical Center); Temeika Fairley (Sister's Network); Dawn Hershman (Columbia University); James Bearden, III (Gibbs Cancer Center); Janice Johnson-Diaz (John Jay College); Antonio Wolff (Johns Hopkins University); Heather Patrick, LaToya Stewart, Portteus Chandini (Susan G Komen for the Cure); Kathleen (Kate) Castro (National Institutes of Health); Florencia Gonzalez (Nueva Vida Inc); Carmen Johnson, (Pink and Black, and the Boston Public Health Commission); Dorothy Gibbons (The Rose Garden); Pink Phurree (Knock Out Roses); Ellen Warner (Sunnybrook Hospital, Toronto, Canada); Laura Esserman, Debby Hamolsky, Michelle Melisko, A Jo Chien (University of California, San Francisco); Virginia Borges (University of Colorado); Susan Domchek (University of Pennsylvania); Dennis Holmes (University of Southern California, Keck School of Medicine, Los Angeles); Alfredo Santillan-Gomez (University of Texas
} 
Health Center, San Antonio); Stacy Lewis (Young Survival Coalition). They also thank Josh Gagne, MA, for his work on this study.

\section{References}

1. Howlader N, Noone A, Krapcho M, et al. SEER Cancer Statistics Review, 1975-2010. Bethesda, MA, 2011.

2. Buchanan N, Roland KB, Rodriguez JL, et al. Opportunities for public health communication, intervention, and future research on breast cancer in younger women. J Women Health. 2013;22:293-298.

3. Ashing-Giwa KT, Padilla G, Tejero J, et al. Understanding the breast cancer experience of women: a qualitative study of African American, Asian American, Latina and Caucasian cancer survivors. Psycho-oncology. 2004;13:408-428.

4. Napoles AM, Ortiz C, O'Brien H, et al. Coping resources and selfrated health among Latina breast cancer survivors. Oncology Nurs Forum. 2011;38:523-531.

5. Janz NK, Mujahid MS, Hawley ST, et al. Racial/ethnic differences in quality of life after diagnosis of breast cancer. J Cancer Surviv. 2009;3:212-222.

6. Graves KD, Jensen RE, Canar J, et al. Through the lens of culture: quality of life among Latina breast cancer survivors. Breast Cancer Res Treat. 2012;136:603-613.

7. Sheppard VB, Harper FW, Davis K, et al. The importance of contextual factors and age in association with anxiety and depression in Black breast cancer patients. Psychooncology. 2014;23:143-150.

8. Ruddy KJ, Greaney ML, Sprunck-Harrild K, et al. Young women with breast cancer: a focus group study of needs and potential interventions. J Adolesc Young Adult Oncol. 2013;2:153-160.

9. Crabtree BF, Miller WL, eds. Doing qualitative research. 2nd ed. Thousand Oaks, CA: Sage Publications; 1999;179-194.

10. Levine EG, Yoo G, Aviv C, et al. Ethnicity and spirituality in breast cancer survivors. J Cancer Surv. 2007;1:212-225.

11. Levine EG, Aviv C, Yoo G, et al. The benefits of prayer on mood and well-being of breast cancer survivors. Support Care Cancer. 2009;17:295-306.

12. Cotton SP, Levine EG, Fitzpatrick CM, et al. Exploring the relationships among spiritual well-being, quality of life, and psychological adjustment in women with breast cancer. Psychooncology. 1999;8:429-438.

13. Lewis PE, Sheng M, Rhodes MM, et al. Psychosocial concerns of young African American breast cancer survivors. J Psychosoc Oncol. 2012;30:168-184.

14. Rodriguez EM, Bowie JV, Frattaroli S, et al. A qualitative exploration of the community partner experience in a faith-based breast cancer educational intervention. Health Educ Res. 2009; 24:760-771.

15. Matthews AK, Berrios N, Darnell JS, et al. A qualitative evaluation of a faith-based breast and cervical cancer screening intervention for African American women. Health Educ Behav. 2006;33:643-663.

16. Tate JD. The role of spirituality in the breast cancer experiences of African American women. J Holist Nurs. 2011;29:249-255.

17. Jones B, Volker DL, Vinerajas $Y$, et al. The meaning of surviving cancer for Latino adolescents and emerging young adults. Cancer Nurs. 2010;33:74-81.

18. Snell L, McCarthy C, Klassen A, et al. Clarifying the expectations of patients undergoing implant breast reconstruction: a qualitative study. Plast Reconstr Surg. 2010;126:1825-1830.

19. Ditsch N, Bauerfeind I, Vodermaier A, et al. A retrospective investigation of women's experience with breast reconstruction after mastectomy. Arch Gynecol Obstet. 2013;287:555-561.

20. Rosenberg SM, Tamimi RM, Gelber S, et al. Body image in recently diagnosed young women with early breast cancer. Psychooncology. 2013;22:1849-1855.

21. Hwang ES, Lichtensztajn DY, Gomez SL, et al. Survival after lumpectomy and mastectomy for early stage invasive breast cancer: the effect of age and hormone receptor status. Cancer. 2013;119:1402-1411.

22. Ruddy KJ, Gelber S, Ginsburg ES, et al. Menopausal symptoms and fertility concerns in premenopausal breast cancer survivors: a comparison to age- and gravidity-matched controls. Menopause. 2011;18:105-108.

23. Partridge AH, Gelber S, Peppercorn J, et al. Web-based survey of fertility issues in young women with breast cancer. J Clin Oncol. 2004;22:4174-4183.

24. Thewes B, Meiser B, Taylor A, et al. Fertility- and menopause-related information needs of younger women with a diagnosis of early breast cancer. J Clin Oncol. 2005;23:5155-5165.

25. Wilkes S, Coulson S, Crosland A, et al. Experience of fertility preservation among younger people diagnosed with cancer. Human Fertil (Camb). 2010;13:151-158.

26. Kirkman M, Stern C, Neil S, et al. Fertility management after breast cancer diagnosis: a qualitative investigation of women's experiences of and recommendations for professional care. Health Care Women Int. 2013;34:50-67.

27. Lee SJ, Schover LR, Partridge AH, et al. American Society of Clinical Oncology recommendations on fertility preservation in cancer patients. J Clin Oncol. 2006;24:2917-2931.

28. Loren AW, Mangu PB, Beck LN, et al. Fertility preservation for patients with cancer: American Society of Clinical Oncology clinical practice guideline update. J Clin Oncol. 2013; 31:2500-2510.

29. Brown A. In US, average retirement age up to 61. Gallup website. http://www.gallup.com/poll/162560/average-retirement-age.aspx. Published May 15, 2013. Accessed September 8, 2015.

30. Adler NE, Page AE, eds. Cancer care for the whole patient: meeting psychosocial health needs. Washington, DC, National Academies Press. http://www.ncbi.nlm.nih.gov/books/NBK4015/. Published 2008. Accessed September 8, 2015.

31. Carey ML, Clinton-McHarg T, Sanson-Fisher RW, et al. Development of cancer needs questionnaire for parents and carers of adolescents and young adults with cancer. Support Care Cancer. 2012;20:991-1010.

32. Patterson P, Pearce A, Slawitschka E: The initial development of an instrument to assess the psychosocial needs and unmet needs of young people who have a parent with cancer: piloting the offspring cancer needs instrument (OCNI). Support Care Cancer. 2011;19:1165-1174

33. Hodgkinson K, Butow P, Hunt GE, et al. The development and evaluation of a measure to assess cancer survivors' unmet supportive care needs: the CaSUN (Cancer Survivors'Unmet Needs measure). Psychooncology. 2007;16:796-804. 